\section{Hipoxemia recurrente y formen oval permeable. Unidad diagnóstica fácilmente inadvertida. Caso clínico}

\author{
FRANCO APPIANI ${ }^{\mathrm{a}}$, POLENTZI URIARTE ${ }^{1,2}$, ROSARIO FERNÁNDEZ $^{\mathrm{b}}$, \\ VICTOR PARRA ${ }^{2}$, FERNANDO PINEDA $^{1,2}$
}

\section{Recurrent hypoxemia secondary to a patent foramen ovale. Report of one case}

Patent foramen ovale (PFO) is a prevalent congenital septal atrial defect usually without pathological significance. In certain pathogenic situations, PFO can trigger episodes of recurrent hypoxemia, a specific condition known as platipnea-orthodeoxia syndrome (POS). We report a 73 years old female presenting with dyspnea and low arterial oxygen saturation. On admission the patient had a hemoglobin saturation of $81 \%$ and an arterial oxygen partial pressure of $50 \mathrm{mmHg}$. After breathing 100\% a 17\% arterial-venous shunt was found (normal: less than 12\%). A transesophageal echocardiogram and a cardiac catheterization showed the presence of a PFO. A percutaneous closure was performed.

(Rev Med Chile 2020; 148: 263-267)

Key words: Cyanosis; Foramen Ovale, Patent; Heart Septal Defects, Atrial; Hepatopulmonary Syndrome.

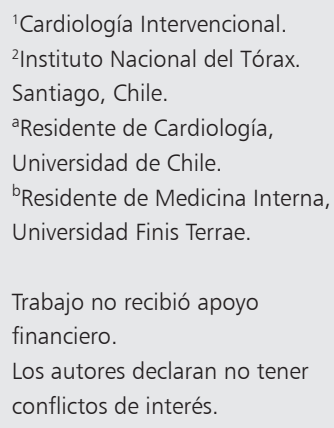

E I foramen oval permeable (FOP) constituye un defecto septal congénito prevalente en la población. Su hallazgo tiende a no tener correlato patológico, pero en ciertas condiciones específicas podría desencadenar hipoxemia recurrente $^{1}$. A continuación relatamos un caso clínico que ilustra las dificultades asociadas al diagnóstico, los fenómenos patogénicos asociados y sus posibilidades terapéuticas actuales.

\section{Caso}

Mujer de 73 años, con antecedentes de hipertensión arterial, cardiopatía coronaria y amaurosis bilateral secundaria a retinitis pigmentosa.

Presenta cuadro de dos meses de disnea de esfuerzo, en múltiples evaluaciones clínicas se demuestra desaturación de oxígeno arterial, siendo tratada como infecciones respiratorias bajas sin éxito.
Al momento del ingreso, consulta por disnea asociada a un episodio de lipotimia. Se observa tranquila, eupneica, en buenas condiciones, hemodinámicamente estable, pero con saturación de oxígeno ambiental de $81 \%$ concordante con hipoxemia arterial en sus exámenes $\left(\mathrm{PO}_{2} 50 \mathrm{mmHg}\right)$. El resto del examen físico cardiovascular y pulmonar se encuentra sin hallazgos patológicos. Inicialmente se observa nula mejoría al oxígeno suplementario situación que resuelve a los pocos minutos tras posicionar al paciente en decúbito.

Los exámenes de laboratorio muestran hemoglobina $14,5 \mathrm{~g} / \mathrm{dl}$, glóbulos blancos $5.200 \mathrm{~mm}^{3}$, pruebas hepáticas normales, creatininemia 0,8 $\mathrm{mg} / \mathrm{dl}$, NT-proBNP $30 \mathrm{pg} / \mathrm{ml}(\mathrm{N}<50)$, dímero D $300 \mathrm{mg} / \mathrm{ml}(\mathrm{N}<500)$ y diferencia $\mathrm{A}-\mathrm{a}$ de $\mathrm{O}_{2}$ de $220 \mathrm{mmHg}(\mathrm{N}<20 \mathrm{mmHg})$. El electrocardiograma, radiografía de tórax y angio-TAC de tórax, sin hallazgos patológicos.

Ante la presencia de insuficiencia respiratoria sin etiología evidente se realiza estudio de corto- 


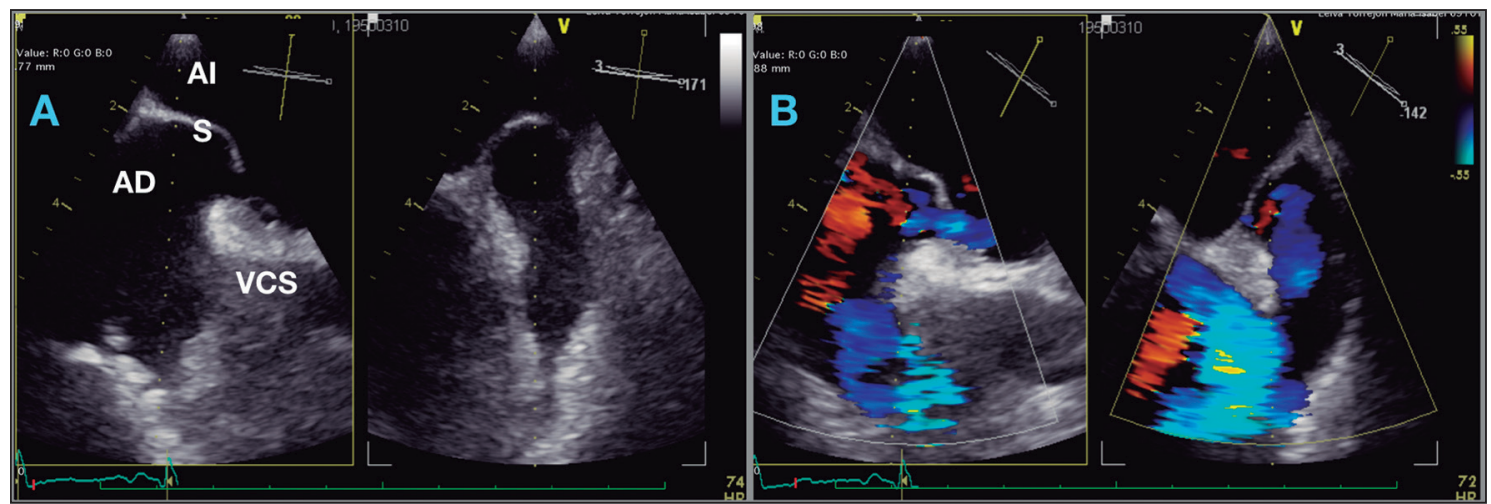

Figura 1. Ecocardiograma Transesofagico con FOP. En la imagen A se aprecia a izquierda visión bicava con su plano ortogonal a derecha. Se observa septum interauricular fino, aneurismático (excursión de $15 \mathrm{~mm}$ ), con presencia de FOP con $6 \mathrm{~mm}$ de ancho de separación y, a través de ésta, flujo bidireccional al Doppler color, predominante de derecha a izquierda (panel B), compatible con FOP. AD: Aurícula derecha; Al: Aurícula izquierda; S: Septum interauricular; VCS: Vena cava superior.

Tabla 1. Valores del cateterismo cardiaco

\begin{tabular}{|lcc|}
\hline Lugar & $\begin{array}{c}\text { Presión } \\
\text { (mmHg) }\end{array}$ & $\begin{array}{c}\text { Saturación } \\
\text { de } \mathbf{O}_{\mathbf{2}} \text { (\%) }\end{array}$ \\
\hline Vena cava superior & 8 & 68 \\
Aurícula derecha & 8 & 71 \\
Ventrículo derecho & 10 & 70 \\
\hline Arteria pulmonar (ID) & $32 / 12(19)$ & 71 \\
Capilar pulmonar & 12 & \\
Vena pulmonar (SI) & & 97 \\
Ventriculo izquierdo & $120 / 10$ & 91 \\
Aorta & $120 / 70$ & 91 \\
Parámetro & Valor & \\
Gasto cardiaco & 7 L/min (Fick) \\
Resistencia vascular pulmonar & 1,0 UW \\
Resistencia vascular sistémica & 11,2 UW \\
\hline
\end{tabular}

ID: inferior derecha; SI: superior izquierda; UW: unidades Wood.

circuito (o shunt) con administración de oxígeno al 100\% y análisis de gasometría arteriovenosa. Los resultados revelan un cortocircuito de $17 \%$ $(\mathrm{N}<12 \%)$.

El ecocardiograma transtorácico muestra dilatación leve de raíz aórtica con insuficiencia leve. Se realiza prueba de solución salina agitada con paso masivo de burbujas al segundo latido a cavidades izquierdas, compatible con cortocircuito intracardiaco de derecha a izquierda. Se complementa estudio con ecocardiograma transesofágico que demuestra un FOP con flujo al doppler color de derecha a izquierda (Figura 1). El sondeo cardíaco derecho e izquierdo revela presiones intracavitarias y gasto cardíaco normal. Estudio de shunt demuestra una relación Qp:Qs de 0,9, por cortocircuito a nivel auricular de derecha a izquierda (Tabla 1).

Los hallazgos son compatibles con síndrome platipnea-ortodeoxia (SPO) y dada la sintomatología persistente, se decide cierre percutáneo del FOP (Figura 2).

Inmediatamente posterior al procedimiento, en posición semisentada, se constata saturación de oxígeno de $99 \%$ ambiental.

Posterior al alta la paciente relata buena capacidad funcional y ausencia de disnea, logrando retornar a sus actividades habituales.

\section{Discusión}

Se describe el caso de un paciente con SPO en relación a un FOP, entidades que si bien pueden estar asociadas merecen un análisis individual.

Inicialmente descrito en el año 1949 como "cianosis ortostática", el SPO hace referencia a su presentación clínica clásica caracterizada por disnea e hipoxemia en posición de pie que mejora en posición supina². Si bien su definición 

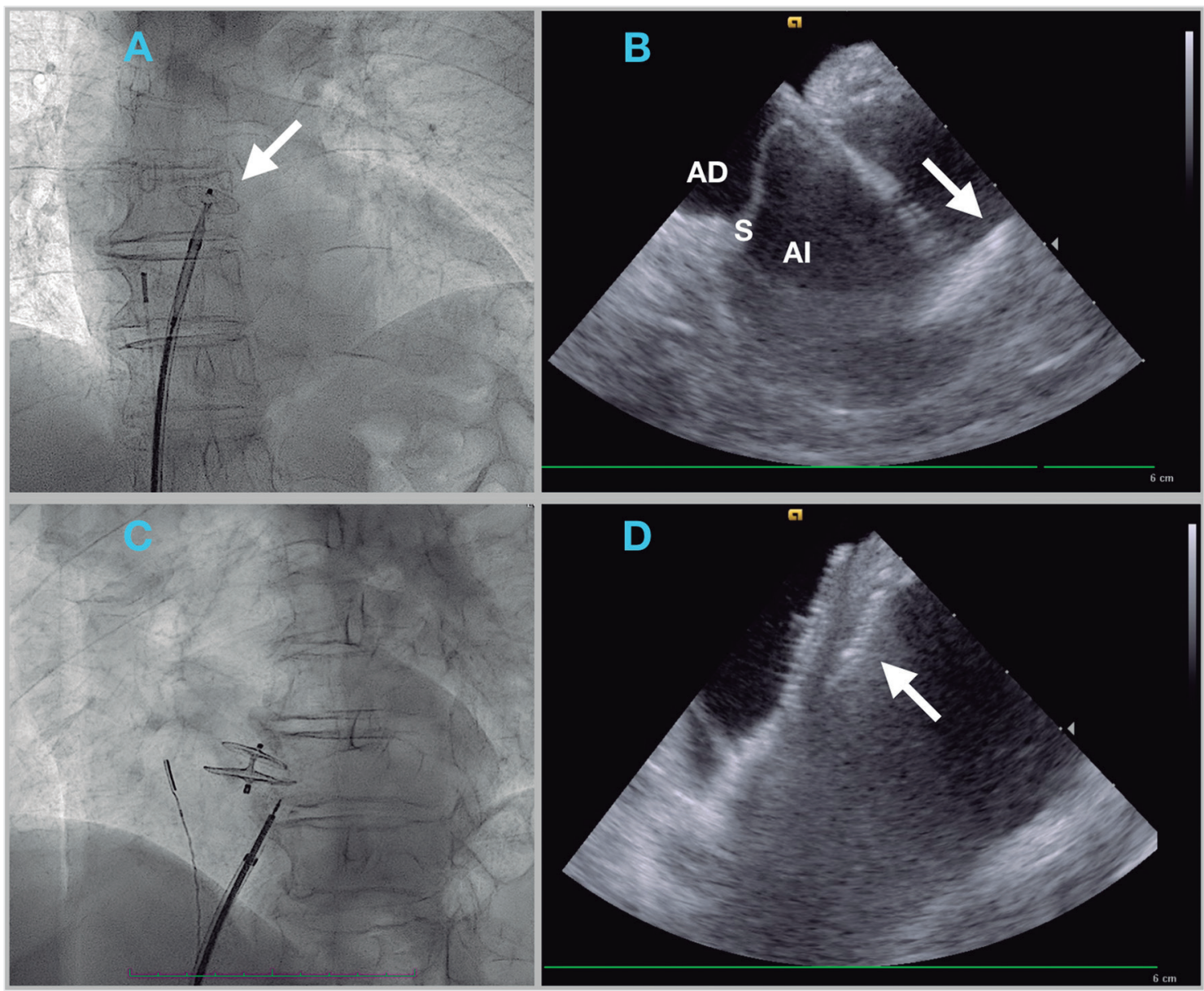

Figura 2. Cierre del FOP con dispositivo percutáneo. En la Imagen A se observa radioscopía torácica anteroposterior con dispositivo de cierre, Amplatzer PFO Ocludder numero 25 (flecha), desplegado aún sin liberación. En la Imagen B se observa visión bajo ecografía intracardiaca simultánea a la imagen anterior. Se aprecia paso de catéter a través del FOP con dispositivo parcialmente desplegado (flecha). Imagen C e Imagen D representan el mismo procedimiento, ahora mostrando dispositivo liberado completamente, con cierre del defecto a nivel del septum interauricular. AD: Aurícula derecha; Al: Aurícula izquierda; S: Septum interauricular.

es sencilla, su diagnóstico habitualmente pasa inadvertido, los pacientes consultan en múltiples oportunidades por insuficiencia respiratoria previo a un diagnóstico definitivo ${ }^{3}$. El SPO se produce tras la mezcla de sangre desoxigenada con sangre oxigenada por paso a través de un cortocircuito. Corresponde a una causa de insuficiencia respiratoria hipoxémica, pero que en forma particular cursa con escasa mejoría con oxigenoterapia. La exposición a oxígeno a 100\% y el consiguiente análisis de la cantidad de oxígeno arteriovenoso, permite objetivar la fracción de shunt específica ${ }^{1}$, como lo observado en el caso expuesto.

Las causas de SPO se dividen en cardiacas y extracardiacas, siendo descrito el FOP como la más frecuente $(87 \%)^{1}$. Sin embargo, previo a responsabilizar al FOP, deben descartarse otras causas cardiacas (comunicación interauricular (CIA) y drenaje pulmonar venoso anómalo) y extracardiacas, como shunts arteriovenosos pulmonares (p.e. Síndrome hepatopulmonar, síndrome de Rendu-Osler-Weber) y enfermedad del parénquima pulmonar (p.e. enfermedad pulmonar obstructiva crónica) ${ }^{1}$. 
Al momento del nacimiento, el foramen oval se cierra tras el desplazamiento y unión entre el septum primum y el septum secundum, generando una fosa oval firme y adherida. En $25 \%$ de la población esta unión de capas ocurre de manera incompleta, generando un FOP, o más bien, un foramen con capacidad de apertura ${ }^{1}$. Habitualmente el FOP es un hallazgo sin correlato patológico, sin embargo, en algunos casos se ha asociado a cuadros como migraña con aura recurrente, embolías paradojales y SPO. Es más, en estos escenarios, se discute sobre el potencial beneficio terapéutico de su cierre ${ }^{7}$.

Dado que la presión auricular izquierda es 5 a $8 \mathrm{mmHg}$ mayor a la presión de aurícula derecha, en el FOP, se genera un afrontamiento entre las capas septales, produciendo un cortocircuito de derecha a izquierda que tiende a ser ínfimo o a estar ausente. Ahora bien, si coexiste alguna condición de incremento de presión en la aurícula derecha (p.e. Hipertensión pulmonar o insuficiencia tricúspidea severa), el cortocircuito puede tornarse significativo resultando en hipoxemia clínica y SPO. No obstante, el estudio invasivo de nuestro paciente demostró presiones en cavidades derechas normales. ¿Entonces, cómo podemos justificar el SPO en este contexto? Curiosamente, en ausencia de una gradiente de presión interauricular, también es posible presenciar un cortocircuito de magnitud suficiente para desencadenar un SPO, siendo necesario para esto, la coexistencia de un sustrato anatómico y otro funcional especifico ${ }^{1-3}$. El sustrato anatómico es el defecto en el septum interauricular, por ejemplo CIA o FOP. El sustrato funcional hace referencia a ciertas variaciones anatómicas cardiacas o pulmonares que permiten redirigir el flujo de entrada hacia la aurícula izquierda, pasando a través del defecto septal, sin requerir un gradiente de presión interauricular. Modificación de la arquitectura de la fosa oval por compresión o elongación de la pared posterior auricular (p.e. Dilatación de la raíz aortica), sumado a remanentes embrionarios que hacen más probable la redirección de flujo (p.e. Válvula de Eustaquio prominente), generan un ambiente ideal para un cortocircuito de derecha a izquierda. Este fenómeno se intensifica en posición de pie gracias al ingreso de la vena cava inferior anatómicamente en situación posteroinferior de la aurícula derecha. A través de una revisión sistemática de la literatura, Abhinav identifica los cambios anatómicos más frecuentes asociados a un FOP relacionado a un SPO, destacando dilatación/elongación/aneurisma aórtico (53\%), neumonectomía (29\%), parálisis diafragmática (17\%) y válvula de Eustaquio prominente (17\%), entre otros ${ }^{1}$. En todos estos casos se refleja el fenómeno de alteración estructural auricular, elemento fundamental para la génesis del shunt en ausencia de gradiente de presión interauricular.

Para el diagnóstico del FOP asociado a cortocircuito de derecha a izquierda, actualmente se recomiendan métodos directos (ecocardiograma transtorácico [ETT] o transesofágico [ETE]) e indirectos (doppler transcraneal [DTC] $)^{4,5}$. Como primera aproximación es razonable emplear el ETT con test de burbujas (utilizando solución salina agitada) para la detección de shunt de derecha a izquierda (sensibilidad $46 \%$ y especificidad 99\%, comparada con ETE). Esta prueba permite también establecer la intensidad y origen del shunt, siendo intracardiaco si las burbujas pasan a cavidades izquierdas antes del cuarto ciclo. Hay que recordar que el cortocircuito en el FOP varía según condiciones hemodinámicas, posturales y respirofasicas. Por esta razón, ante un resultado no concluyente, la prueba de burbujas podría mejorar su rendimiento con el paciente en posición de pie, implementando maniobra de Valsalva o mediante infusión de burbujas por vena femoral, siendo este último especialmente útil cuando se sospecha SPO por un FOP en relación a una válvula de Eustaquio prominente ${ }^{4}$. La detección de un cortocircuito mejora con el uso de ETE (87\% sensibilidad, $91 \%$ especificidad) o DTC (97\% sensibilidad, 91\% especificidad), siendo el ETE de elección para observar los detalles anatómicos del FOP y otras causas de SPO cardiaco (p.e. CIA) ${ }^{5}$.

La experiencia acumulada en el tratamiento del SPO es escasa. Un estudio observacional reúne 17 pacientes con SPO sometidos a cierre del FOP con dispositivo percutáneo. Solo 11 pacientes (65\%) demostraron mejoría de disnea e hipoxemia. En aquellos donde no se demostró mejoría había una causa pulmonar coexistente que explicaba la hipoxemia ${ }^{6}$. Si no hay indicación quirúrgica por otra razón, el cierre percutáneo es actualmente el tratamiento de elección en centros con experiencia $^{1,2,7}$. El estudio RESPECT, dirigido a evaluar el cierre percutáneo de FOP en pacientes con accidente cerebrovascular criptogénico ( $\mathrm{n}=499$, en rama intervención), evidencia bajas tasas de complicaciones inmediatas, siendo algunas de ellas 
la fibrilación auricular $(1,2 \%)$, hematoma en sitio de punción $(0,2 \%)$, taponamiento pericárdico $(0,4 \%)$, shunt residual $(0,2 \%)$ y PCR $(0,2 \%)^{7}$.

En suma, este trabajo busca mostrar la historia habitual de los pacientes con SPO y FOP. El ETT con test de burbujas es una aproximación inicial razonable y debe ser de rutina en pacientes con hipoxemia no resuelta. El FOP constituye un defecto congénito que solo tendrá implicancia patológica cuando se presenten situaciones generadoras de SPO, como en este caso probablemente lo fue la presencia de dilatación de la raíz aórtica. Actualmente el tratamiento percutáneo conlleva una baja tasa de complicaciones y debe realizarse ante pacientes con sintomatología invalidante. Sin embargo, para asegurar el éxito terapéutico, es importante descartar otras causas de hipoxemia previo al cierre del FOP.

\section{Referencias}

1. Agrawal A, Palkar A, Talwar A. The multiple dimensions of Platypnea-Orthodeoxia syndrome: A review. Respir
Med 2017; 129: 31-8.

2. De Vecchis R, Baldi C, Ariano C, Giasi A, Cioppa C. Platypnea-orthodeoxia syndrome : Orthostatic dyspnea and possible pathophysiological substrates. Herz 2017; 42 (4): 384-9.

3. Mojadidi MK, Ruiz JC, Chertoff J, Zaman MO, Elgendy IY, et al. Patent Foramen Ovale and Hypoxemia. Cardiol Rev 2019; 27 (1): 34-40.

4. Mahmoud AN, Elgendy IY, Agarwal N, Tobis JM, Mojadidi MK. Identification and Quantification of Patent Foramen Ovale-Mediated Shunts: Echocardiography and Transcranial Doppler. Interv Cardiol Clin 2017; 6 (4): 495-504.

5. Puchalski M, Lui G, Miller-Hance W, Brook M, Young L, Bhat A. Guidelines for Performing a Comprehensive Transesophageal Echocardiographic. J Am Soc Echocardiogr 2019; 32 (2): 173-215.

6. Collado FMS, Poulin MF, Murphy JJ, Jneid H, Kavinsky CJ. Patent Foramen Ovale Closure for Stroke Prevention and Other Disorders. J Am Heart Assoc 2018; 7 (12).

7. Mojadidi MK, Gevorgyan R, Noureddin N, Tobis JM. The effect of patent foramen ovale closure in patients with platypnea-orthodeoxia syndrome. Catheter Cardiovasc Interv 2015; 86: 701-7. 